\title{
Neutron emission in electromagnetic dissociation of ultrarelativistic $\mathrm{Pb}$ ions
}

\author{
M. B. Golubeva, F. F. Guber, T. L. Karavicheva, ${ }^{*}$ E. V. Karpechev, A. B. Kurepin, A. I. Maevskaya, I. A. Pshenichnov, ${ }^{\dagger}$ \\ A. I. Reshetin, K. A. Shileev, V. V. Tiflov, and N. S. Topilskaya \\ Institute for Nuclear Research, Russian Academy of Science, 117312 Moscow, Russia \\ P. Szymanski \\ CERN, EP Division, CH-1211, Geneva 23, Switzerland, and Soltan Institute for Nuclear Studies, Warsaw, Poland \\ I. Efthymiopoulos and L. Gatignon \\ CERN, AB/ATB Division, CH-1211, Geneva 23, Switzerland \\ P. Cortese and G. Dellacasa \\ Dipartimento di Scienze e Tecnologie Avanzate and INFN, Corso Borsalino 54, I-15100 Alessandria, Italy
}

R. Arnaldi, N. De Marco, A. Ferretti, M. Gallio, A. Musso, C. Oppedisano, A. Piccotti, E. Scomparin, and E. Vercellin Università di Torino and INFN, Via Pietro Giuria 1, I-10125 Torino, Italy

C. Cicalò, G. Puddu, and E. Siddi

Università di Cagliari and INFN, Casella Postale 170, I-09042 Monserrato (Cagliari), Italy

(Received 17 December 2003; revised manuscript received 23 July 2004; published 24 February 2005)

\begin{abstract}
New data on forward neutron emission in fragmentation of $30 A-\mathrm{GeV}^{208} \mathrm{~Pb}$ ions on $\mathrm{Al}, \mathrm{Cu}, \mathrm{Sn}$, and $\mathrm{Pb}$ nuclei are presented. The measurements were performed at the CERN SPS in the framework of the ALICE-LUMI experiment. The measured cross sections are compared with predictions of the RELDIS model for electromagnetic interactions and with results of the abrasion-ablation model for hadronic interactions. The electromagnetic excitation of a $\mathrm{Pb}$ projectile followed by single- and double-neutron emission is found to be the dominant process in full agreement with theoretical estimations. The measured $1 n X$ cross sections are generally well described. The measured $2 n X$ data, which are 4-5 times lower than the $1 n X$ data, are slightly underestimated by theory. Nevertheless, the sum of $1 n X$ and $2 n X$ cross sections is in good agreement with theory. This confirms the predictive power of the RELDIS model, which can be used to calculate the sum of $1 n X$ and $2 n X$ emission rates for the purpose of calibration of luminosity measurements in $\mathrm{PbPb}$ collisions at the Large Hadron Collider at CERN.
\end{abstract}

DOI: 10.1103/PhysRevC.71.024905

PACS number(s): 25.75.-q, 25.70.De, 29.27.-a

\section{INTRODUCTION}

The main goal of the ultrarelativistic heavy-ion collision programs at the CERN Super Proton Synchrotron (SPS), the Relativistic Heavy Ion Collider (RHIC), and the future Large Hadron Collider (LHC) at CERN is to study hot and dense nuclear matter created in such collisions. The efforts are focused on searching for signals of phase transitions between hadronic and quark-gluon phases [1-3]. The latter state of nuclear matter is expected to be formed in ultrarelativistic nuclear collisions with large overlap of collision partners. At ultrarelativistic energies a projectile nucleus with the Lorentz factor $\gamma \gg 1$ can be thought of as a Lorentz-contracted sheet of nuclear matter.

The Coulomb fields of colliding nuclei are also highly Lorentz-contracted. A simple estimate shows that, during a short time when nuclei are close to each other, the potential of

\footnotetext{
*Corresponding author: tatiana@inr.ru.

†Present address: Frankfurt Institute for Advanced Studies, J.-W. Goethe University, D-60054 Frankfurt am Main, Germany.
}

the Lorentz-boosted Coulomb field of a partner with charge $Z$ is very strong, $V_{c} \sim \alpha \gamma Z / b$, where $b$ is an impact parameter and $\alpha$ is the fine structure constant. Indeed, for typical values at the CERN SPS $-\gamma \alpha \sim 1, Z \sim 80$, and $b \sim 15 \mathrm{fm}$ - this potential exceeds the effective nucleon-nucleus potential of $\sim 50 \mathrm{MeV}$. Therefore, one or both nuclei may be disintegrated by the long-range electromagnetic forces in ultraperipheral collisions without direct overlap of nuclear densities, at $b \geqslant R_{1}+R_{2}$, where $R_{1}$ and $R_{2}$ are the nuclear radii. This phenomenon, known under the name of electromagnetic dissociation (ED) [4,5], allows us to study the behavior of nuclear matter under a short-term impact of very strong electromagnetic fields.

The ED process plays an important role in heavy-ion colliders. In the rest frame of a colliding nucleus the Lorentz factor of another nucleus is given by $\gamma=2 \gamma_{\text {beam }}^{2}-1$, where $\gamma_{\text {beam }}$ is the Lorentz factor for each heavy-ion beam in the laboratory system. For the LHC at CERN, which is expected to become operational with $\mathrm{Pb}$ beams in $2008, \gamma \sim 1.7 \times 10^{7}$ and the Lorentz contraction of the Coulomb field of $\mathrm{Pb}$ nuclei in $\mathrm{PbPb}$ collisions will become tremendous.

A set of theoretical predictions has already been made for ultraperipheral $\mathrm{PbPb}$ collisions at the LHC [4-10]. Several 

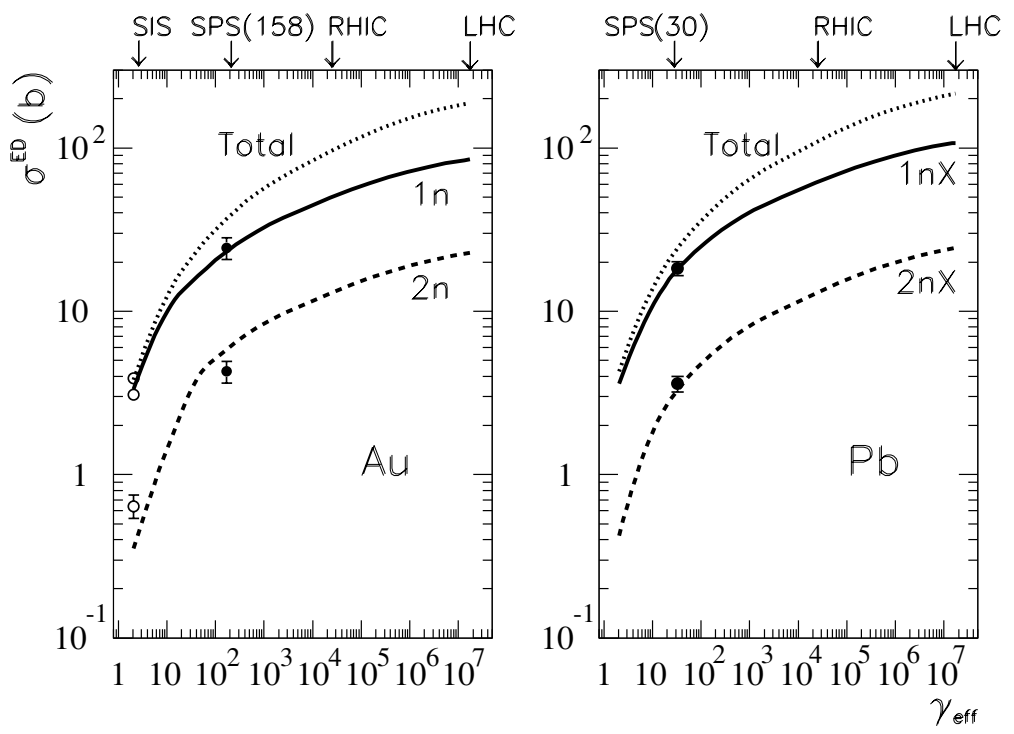

FIG. 1. (Left) Measured $1 n$ and $2 n$ ED cross sections for $\mathrm{Au}$ electromagnetic dissociation are shown by open circles (Ref. [16], AuAu collisions) and filled circles (Ref. [17], AuPb collision data rescaled to $\mathrm{AuAu}$ case). A datum for the total ED cross section of Ref. [16] is also shown. (Right) Measured $1 n X$ and $2 n X$ ED cross sections for $\mathrm{PbPb}$ collisions (present work) are shown by filled circles. Neutrons can be accompanied by other particles denoted as $X$. RELDIS model results for the total, $1 n$, and $2 n$ (left, $\mathrm{AuAu}$ ) and the total, $1 n X$, and $2 n X$ (right, $\mathrm{PbPb}$ ) ED cross sections are shown by dotted, solid, and dashed lines, respectively.

topics include, for example, multiple $e^{+} e^{-}$production and exotic particle production in electromagnetic processes [5,7], multifragmentation of nuclei by strong electromagnetic fields [8], and multiple excitations of giant resonances in colliding nuclei $[6,7,9,10]$.

Since the ED cross section in $\mathrm{PbPb}$ collisions becomes very large ( 200 b) $[9,11]$, it imposes severe restrictions on beam lifetime at the LHC [11]. The initial charge-to-mass ratio of $\mathrm{Pb}$ ions and, consequently, their magnetic rigidity are changed due to electromagnetic dissociation and $e^{+} e^{-}$production accompanied by electron capture [11]. Both phenomena lead to beam loss. Specifically, since single-neutron emission dominates in the ED process, the residual $A-1 \mathrm{~Pb}$ nuclei will impact on a localized zone along the beam pipe, leading to local heating [12]. However, because correlated forward-backward neutron emission in mutual electromagnetic dissociation will be used for beam luminosity monitoring [13-15], precise knowledge of the ED cross sections for specific neutron emission channels is highly desirable. Therefore there exists the need to extensively test the theoretical models with high-quality experimental data.

The question arises whether there are enough experimental data to verify theoretical estimations. In particular, to what extent do $\mathrm{Au}$ electromagnetic dissociation data serve this purpose? Presently there exist single dissociation data for $\mathrm{Au}$ nuclei obtained at GSI SIS [16] and at CERN SPS [17] and mutual dissociation data recently obtained at RHIC [18].

Neutron emission channels of $\mathrm{Au}$ dissociation have been studied by activation methods at $1 A \mathrm{GeV}[16]$ and at $158 A \mathrm{GeV}$ [17] in fixed-target experiments. As confirmed by calculations [9], the cross-section data on $\mathrm{Au}$ dissociation by $158 \mathrm{~A}-\mathrm{GeV}$ $\mathrm{Pb}$ ions [17] may be well described by the RELDIS model based on the Weizsäcker-Williams (WW) method of equivalent photons. A small contribution due to hadronic interactions should be additionally taken into account to describe the data on ${ }^{197} \mathrm{Au} \rightarrow{ }^{196} \mathrm{Au}+n$ and ${ }^{197} \mathrm{Au} \rightarrow{ }^{195} \mathrm{Au}+2 n$ reactions. The mentioned data and calculation results for ED cross sections are visualized in Fig. 1. As shown, the $1 n$ cross section at
$1 A \mathrm{GeV}$ is well described, whereas the $2 n$ value is underestimated by theory [9].

The ratios of $1 n$ and $2 n$ emission cross sections of mutual electromagnetic dissociation in $(60+60) A-\mathrm{GeV} \mathrm{AuAu}$ collisions were recently measured at RHIC [18]. The ratios of these ED cross sections to the total reaction cross section were also obtained. Good agreement with theoretical ratios $[6,9]$ was found. However, absolute cross sections for specific dissociation channels were not reported in Ref. [18].

Despite the similarity in mass, the differences in nuclear structure of $\mathrm{Pb}$ and $\mathrm{Au}$, namely the excitation parameters of giant dipole resonance (GDR), the nuclear level densities of initial and final nuclei in neutron emission channels are noticeable. Indeed, $\mathrm{Pb}$ is a double-magic closed-shell nucleus, whereas $\mathrm{Au}$ is not. This makes it difficult to use the data on $\mathrm{Au}$ dissociation instead of $\mathrm{Pb}$ data to estimate precisely the rates of the beam loss resulting from the ED process. For example, the total ED cross section for $(100+100) A-G e V$ AuAu collisions is equal to $95 \mathrm{~b}$ according to the RELDIS model, whereas it is estimated as $108 \mathrm{~b}$ for $\mathrm{PbPb}$ collisions at the same energy. In contrast, the energy dependence of the ED cross sections (see Fig. 1) is well known from the WW method. Therefore, testing the RELDIS model with experimental data on $\mathrm{Pb}$ dissociation at lower energies makes it possible to use the model to estimate the ED cross sections for $\mathrm{PbPb}$ collisions at the LHC.

To our knowledge, there are no absolute cross-section data obtained via direct detection of neutrons from electromagnetic dissociation of lead nuclei. The aim of the present paper is to report the cross sections of neutron emission in electromagnetic dissociation of $30 \mathrm{~A}-\mathrm{GeV}$ lead nuclei on $\mathrm{Al}, \mathrm{Cu}, \mathrm{Sn}$, and $\mathrm{Pb}$ targets. The measurements were performed at the $\mathrm{H} 8$ beam line of the CERN SPS in the framework of the ALICE-LUMI experiment. The obtained data close the gap in experimental information on electromagnetic dissociation of ultrarelativistic $\mathrm{Pb}$ nuclei. Previously, only the total dissociation cross sections of $158 \mathrm{~A}-\mathrm{GeV} \mathrm{Pb}$ ions (including the hadronic contribution) have been measured $[19,20]$. Other groups have recently reported [21-23] charge-changing cross sections for the same ions. 


\section{THEORETICAL CONCEPTS OF Pb DISSOCIATION}

Before proceeding with a description of the experiment and obtained results, we recall here the basic theoretical concepts widely used to consider electromagnetic dissociation of nuclei at ultrarelativistic energies.

\section{A. Electromagnetic dissociation}

Ultraperipheral heavy-ion collisions without direct overlap of nuclear densities can be considered within the WW method of equivalent (virtual) photons [4,5,7]. These photons induce photonuclear reactions, leading to emission of neutrons among other particles. Since the flux of equivalent photons, as seen by a projectile, is proportional to the square of the target charge, one can expect an important electromagnetic contribution to the projectile dissociation cross section for medium and heavy target nuclei.

A detailed description of the RELDIS model for electromagnetic dissociation of nuclei was given in Ref. [9]. In addition to single-photon absorption, by adopting the harmonicoscillator picture of multiple excitations in combination with the folding model [24,25], double-photon absorption is also accounted for in the RELDIS model.

The energy-integrated convolution of the equivalent photon spectra and the total photoabsorption cross section serves as a key ingredient in calculations providing the total ED cross section [9]. The total photoabsorption cross section for $\mathrm{Pb}$ is taken from the Lorentz curve fits with the parameters of Ref. [26] corrected according to the prescription of Ref. [27].

Branching ratios for neutron emission in $\gamma \mathrm{Pb}$ reactions are calculated by means of the cascade-evaporation-fissionmultifragmentation model of photonuclear reactions [28,29]. When a nucleus absorbs one or two virtual photons in the giant resonance region $6 \leqslant E_{\gamma} \leqslant 30 \mathrm{MeV}$, their energies are completely transformed into excitation energy of this nucleus. GDR excitation in $\mathrm{Pb}$ by equivalent photons and subsequent decay of an excited compound nucleus give the main contribution to $1 n$ and $2 n$ emission. In our ED calculations only evaporation-fission competition matters for compound nucleus decay, since low-energy photons dominate in the spectrum of equivalent photons [25]. Calculated photonuclear $1 n$ and $2 n$ cross sections for $\mathrm{Pb}$ are plotted in Fig. 2 for comparison with the experimental data of Refs. [30-33]. By invoking an empirical procedure proposed in Ref. [27], Saclay [30] and Livermore [31] data for $1 n$ emission can be put in agreement. These data are well described by the RELDIS model. However, the $2 n$ data from these two laboratories are inconsistent with each other both in shape and normalization up to $\sim 50 \%$. We mention that the RELDIS results are closer to the Saclay data [30].

In the rest frame of an excited nucleus the kinetic-energy spectrum of evaporated neutrons is very soft. Most of the neutrons are emitted isotropically and have kinetic energies below $3 \mathrm{MeV}$. Therefore, in the laboratory system neutrons are emitted almost with the rapidity of the initial $\mathrm{Pb}$ beam. Transverse momentum distributions of neutrons in the laboratory frame are shown in Fig. 3 separately for $1 n$ and $2 n$ emission channels. In the latter case the distributions for

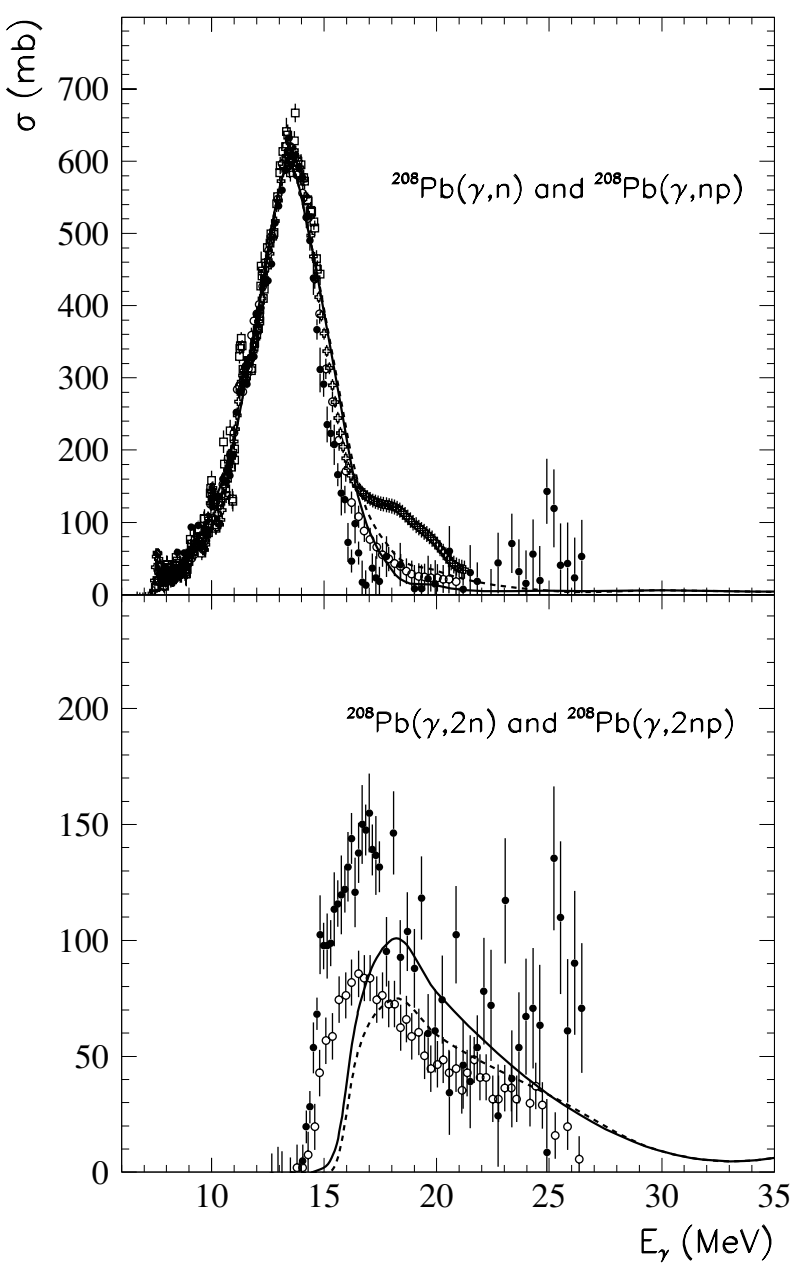

FIG. 2. Photonuclear $1 n$ and $2 n$ cross sections for lead. Open and closed circles are Saclay [30] and Livermore [31] data, respectively, rescaled according to Ref. [27]. Crosses, Saratov data [32]; squares, Moscow evaluated data [33]. The results of the RELDIS model are given by dashed and solid lines for the variants with and without inclusion of a $25 \%$ contribution of direct nonstatistical $1 n$ emission, respectively.

the first and second emitted neutrons are shown separately. Following the usual description of compound nucleus decay $[8,9,25]$, neutron evaporation is considered as a slow process of sequential neutron emission events. Therefore, the first and second emitted neutrons are distinguishable in our model. Since a part of the initial excitation energy in $2 n$ events is already removed by the first evaporated neutron, the second one has lower kinetic energy on average. This is reflected in a narrower $P_{t}$ distribution for such neutrons.

However, the statistical decay of an excited compound nucleus is not the only process responsible for neutron emission. Very early studies [34,35] devoted to the measurements of the neutron spectra in photoabsorption have demonstrated an excess of fast neutrons with kinetic energy $\geqslant 4 \mathrm{MeV}$ compared to the predictions of the statistical evaporation model. This was attributed to the direct emission of neutrons in photoabsoprtion. After such direct emission of a single neutron, the emission of a second neutron is generally 


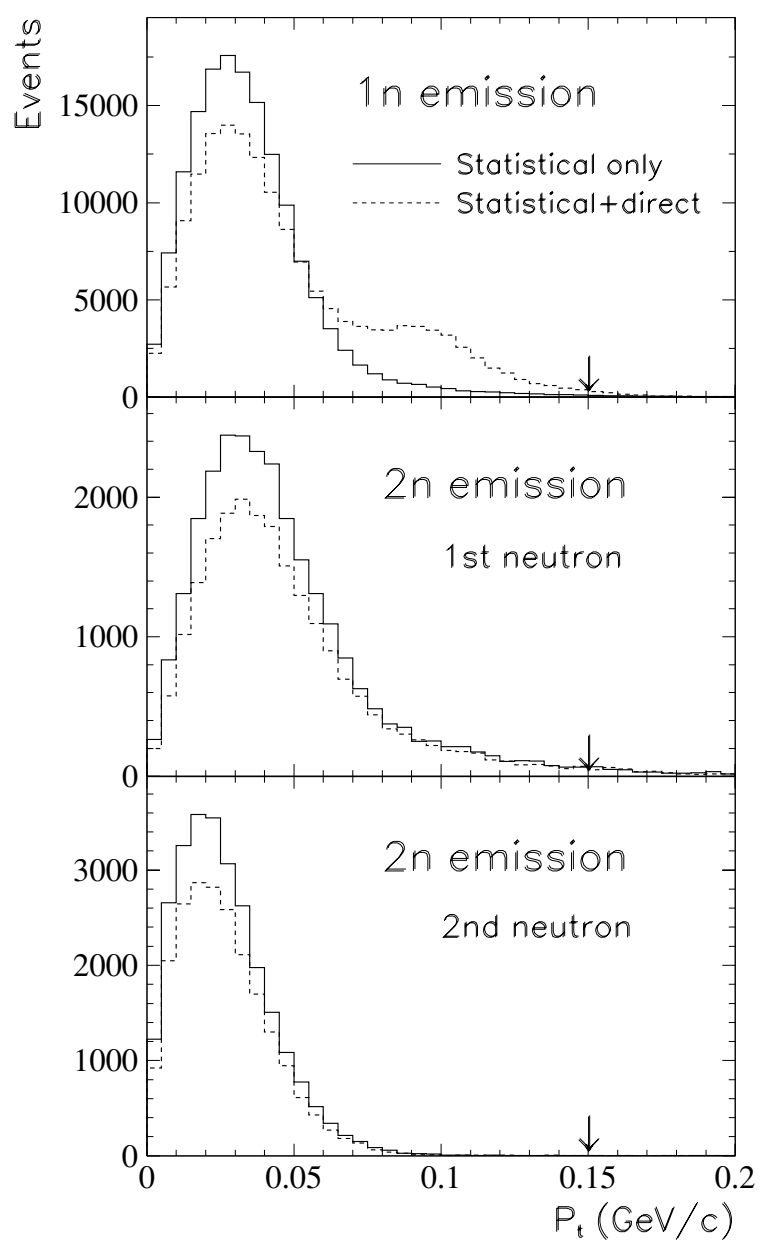

FIG. 3. Distributions of transverse momentum of neutrons emitted in electromagnetic dissociation of $\mathrm{Pb}$ nuclei as predicted by the RELDIS model for $1 n$ and $2 n$ emission. The results for pure statistical decay are given by solid histograms; those for statistical decay with a $25 \%$ admixture of direct neutron emission are given by dashed histograms. The acceptance limit of the present measurements is marked by arrows.

impossible, even though the initial photon energy exceeds the $2 n$ emission threshold. In such a way the $(\gamma, 2 n)$ channel is slightly suppressed in comparison with the pure statistical decay, as shown in Fig. 2. Evidence of direct neutron emission in photoabsorption on $\mathrm{Au}$ and $\mathrm{Pb}$ nuclei was also given in Ref. [30] based on the analysis of competition between $1 n$ and $2 n$ emission channels. The existence of direct neutron emission in photoabsorption on ${ }^{208} \mathrm{~Pb}$ has been clearly demonstrated in modern studies [36]. The process where a fast nucleon is emitted and the final state ${ }^{207} \mathrm{~Pb}$ nucleus is left with low excitation energy $(\leqslant 3 \mathrm{MeV})$ was experimentally identified [36].

By the above-mentioned studies the contribution of direct neutron emission was estimated as $\sim 10-30 \%$. It strongly depends on the excitation energy of the compound nucleus [36]. We assumed a $25 \%$ admixture of the nonstatistical component in our calculations. The calculated transversemomentum distributions of neutrons emitted in electromagnetic dissociation of lead at $30 \mathrm{~A} \mathrm{GeV}$ are given in Fig. 3 with and without accounting for the nonstatistical contribution of GDR decay. The nonstatistical contribution is clearly visible as a shoulder at $P_{t} \sim 0.1 \mathrm{GeV} / c$ in the $P_{t}$ distribution for $1 n$ emission.

As follows from Fig. 3, in both cases, with and without accounting for the nonstatistical component in electromagnetic dissociation, most of the neutrons are emitted with $P_{t}<$ $0.15 \mathrm{GeV} / c$. Based on this observation we have decided upon the transverse size of the neutron calorimeter and its location with respect to the target.

\section{B. Neutron emission in grazing hadronic interactions}

With respect to few-neutron emission, peripheral (grazing) hadronic heavy-ion collisions can contribute as well. It is important to estimate the $1 n X$ and $2 n X$ emission cross sections in such collisions. This can be done in the framework of the well-known abrasion-ablation model [37-39], which is based on a participant-spectator picture of heavy-ion collisions.

Participants originate from the overlapping parts of the colliding nuclei; their nonoverlapping parts are considered as spectators. Nucleons from the participant zone are separated (abraded) from spectators, which represent excited remnants of the initial nuclei (prefragments). In recent studies [40,41] the excitation energy of prefragments is estimated as $13-26 \mathrm{MeV}$ per each abraded nucleon on average.

Finally, secondary decays of these prefragments take place in what is known as an ablation process [37-39]. The decay of prefragments is treated as evaporation-fission competition with neutrons evaporated among other particles. Therefore, neutrons are produced both in the abrasion and ablation steps, but a noticeable difference is expected in their kinematical characteristics.

In the abrasion step, nucleons are knocked out from the initial nuclei as the result of individual $N N$ collisions accompanied by production of secondary hadrons. The average transverse momentum of such nucleons, $\left\langle P_{t}\right\rangle \sim 0.2-$ $0.4 \mathrm{GeV} / c$ [42], is higher compared to ED neutrons; their $P_{t}$ distributions are shown in Fig. 3. Only neutrons evaporated from excited nuclear fragments in the ablation step have a narrow $P_{t}$ distribution similar to the one shown in Fig. 3 . These neutrons can mimic neutrons emitted in electromagnetic dissociation.

Despite having certain simplifications, the picture of heavy-ion collisions, described here explains quite well the production of nuclear fragments at intermediate energies, $\sim 1 A \mathrm{GeV}[40,41]$. There is a hope that this picture remains valid also at relativistic energies, $\sim 10-100 \mathrm{~A} \mathrm{GeV}$.

\section{Quantitative predictions for neutron emission cross sections}

The results of the RELDIS model for electromagnetic dissociation of $30 A-\mathrm{GeV} \mathrm{Pb}$ on a lead target are presented in Table I for $1 n X, 2 n X, 3 n X, 4 n X$, and $5 n X$ channels. The cross sections of the emission of a given number of neutrons are listed. Neutrons can be accompanied by other particles denoted as $X$. Calculations were performed with and without the admixture of nonstatistical GDR decays. Owing to the 
TABLE I. Calculated cross sections (b) for emission of a given number of neutrons in electromagnetic dissociation and hadronic fragmentation of $30 \mathrm{~A}-\mathrm{GeV} \mathrm{Pb}$ nuclei on a lead target.

\begin{tabular}{lccc}
\hline \hline $\begin{array}{l}\text { Number } \\
\text { of neutrons }\end{array}$ & $\begin{array}{c}\text { ED } \\
\text { (Stat. only) }\end{array}$ & $\begin{array}{c}\text { ED } \\
\text { (Stat. }+25 \% \\
\text { direct) }\end{array}$ & $\begin{array}{c}\text { Hadronic } \\
\text { fragmentation }\end{array}$ \\
\hline $1 n X$ & 18.25 & 19.05 & 0.15 \\
$2 n X$ & 3.27 & 2.66 & 0.16 \\
$3 n X$ & 1.09 & 1.02 & 0.18 \\
$4 n X$ & 0.45 & 0.45 & 0.18 \\
$5 n X$ & 0.24 & 0.24 & 0.10 \\
\hline Total ED & 24.4 & 24.4 & \\
cross section & & & \\
\hline \hline
\end{tabular}

direct nonstatistical emission of a single neutron the $1 n X$ cross section is increased, whereas the $2 n X$ cross section is suppressed compared to the case of pure statistical GDR decay, which is simulated by an evaporation code in our calculations. The dominance of the $1 n$ emission in electromagnetic dissociation is clearly demonstrated by calculations.

The results of our version of the abrasion-ablation model for hadronic fragmentation are also given in Table I. The hadronic fragmentation cross sections are found to be very small compared to the ED cross sections, in particular for $1 n$ and $2 n$ channels. This is not surprising because the geometrical cross sections for the abrasion of one or two nucleons are only $0.1-0.3 \mathrm{~b}$. Indeed, only a thin ring on the low-density nuclear periphery should be involved in collisions that result in the removal of a few nucleons and leave a large exited remnant. Generally, even a moderately excited prefragment evaporates at least one additional neutron, thus reducing the cross section of the $1 n$ channel to $\sim 0.15 \mathrm{~b}$.

The ED cross sections listed in Table I rapidly decrease with an increase in the number of emitted neutrons, contrary to the hadronic cross sections. In Sec. IV the calculated cross sections will be compared with obtained experimental values.

\section{MEASUREMENTS OF Pb DISSOCIATION}

Our experimental setup at the H8 beam line in the north experimental area of the CERN SPS accelerator is shown schematically in Fig. 4. The setup comprises a target system, bending magnets (MBPL and MBPS), a hadron calorimeter, and scintillator counters ( $\mathrm{S} 0, \mathrm{~S} 1$, and $\mathrm{SS})$.

The primary ${ }^{208} \mathrm{~Pb} 30 \mathrm{~A}-\mathrm{GeV}$ beam was focused on a specially installed interaction target. As shown in Fig. 4, charged particles were deflected out of the calorimeter acceptance by the MBPL and MBPS magnets with working fields of 3.8 $\mathrm{T} * \mathrm{~m}$, and $1.9 \mathrm{~T} * \mathrm{~m}$, respectively. These magnets were located close to the target and the primary beam was deflected in the horizontal plane. The deflection distance from the initial beam direction of the incoming $\mathrm{Pb}$ beam amounted to $65.3 \mathrm{~mm}$ at the face of the neutron calorimeter. This made it possible to put the plastic scintillator detector SS with dimensions of $2 \mathrm{~cm} \times 2 \mathrm{~cm}$ in this place.

The SS detector was used to register deflected charged particles and was able to tag the $\mathrm{Pb}$ ions that survive the $4.8-\mathrm{m}$ flight in air. It was used only to tune the beam position and not in the trigger. Forward neutrons from $\mathrm{Pb}$ dissociation were not affected by magnetic fields and were registered by the neutron calorimeter, which was located in the beam direction beyond the magnets.

The target was put between two plastic scintillator detectors, S0 and S1, made of polystyrene-based material with 4-5\% PTP POPOP as shifting dopants. The detectors (S0, $\mathrm{S} 1, \mathrm{SS}$ ) were $2-\mathrm{mm}$-thick plates with the size of $2 \mathrm{~cm} \times 2 \mathrm{~cm}$. All detectors demonstrated a very good energy loss resolution of the order of a few percent. A huge peak caused by incoming $30 A-G e V$ lead ions was clearly visible and was accompanied by small tails at lower energy loss in all detectors. In the case of the S0 detector the tail was due to nuclear fragments presented in the incoming lead ion beam, which originated from $\mathrm{Pb}$ interactions with the surrounding air upstream of the S0 detector. In the case of the S1 and SS detectors the tail was due to nuclear fragments produced in air and in the target.

The incident lead ions were counted by the S0 detector; the $S 1$ detector was used to identify the lead ions that do not suffer fragmentation in air or in the target. Four targets of various materials and sizes listed in Table II were mounted on a ladder, which can be moved horizontally for positioning.

All targets were cylinder shaped with radii of $38 \pm 0.02 \mathrm{~mm}$. Empty-target runs were provided and the background resulting from the neutron emission in dissociation of $\mathrm{Pb}$ nuclei on air molecules, on the material of the beam monitors, and on the beam slits was subtracted from the raw data. These runs were performed following each target changing and beam stop.

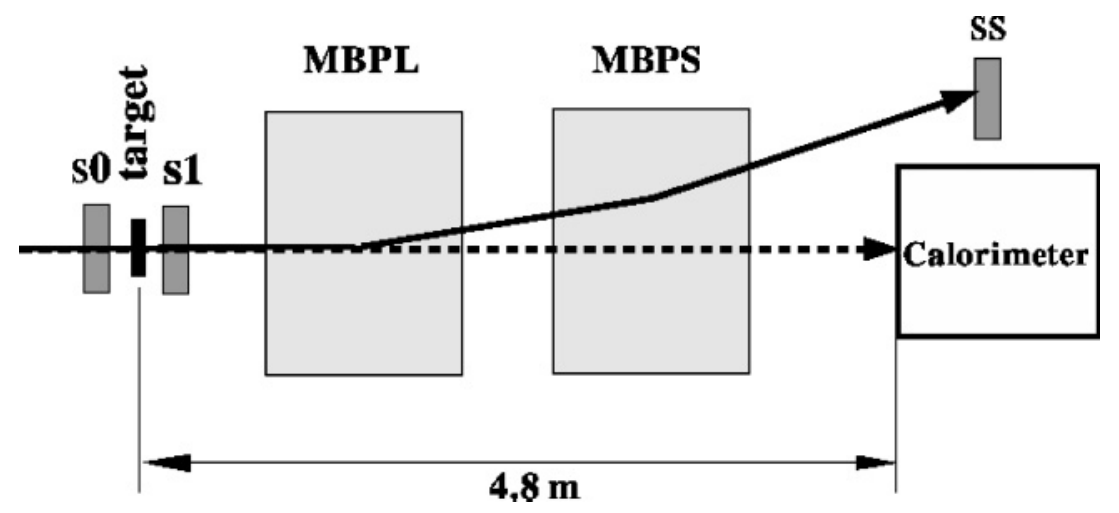

FIG. 4. Schematic view of experimental setup: S0, S1, and SS are plastic scintillator detectors; MBPL and MBPS are magnets. Trajectories of charged particles and neutrons are schematically depicted by solid and dashed lines, respectively. 
TABLE II. Target specifications.

\begin{tabular}{lccc}
\hline $\begin{array}{l}\text { Target } \\
\text { material }\end{array}$ & Weight $(\mathrm{g})$ & Thickness $(\mathrm{mm})$ & $\begin{array}{r}\text { Density } \\
\left(\mathrm{g} / \mathrm{cm}^{3}\right)\end{array}$ \\
\hline $\mathrm{Al}$ & $45.28 \pm 0.01$ & $14.80 \pm 0.02$ & 2.70 \\
$\mathrm{Cu}$ & $45.02 \pm 0.01$ & $4.50 \pm 0.02$ & 8.96 \\
$\mathrm{Sn}$ & $28.03 \pm 0.01$ & $3.40 \pm 0.02$ & 7.31 \\
$\mathrm{~Pb}$ & $37.31 \pm 0.01$ & $2.94 \pm 0.02$ & 11.35 \\
\hline \hline
\end{tabular}

The acceptance limit for transverse momentum of neutrons is shown in Fig. 3. As shown by Monte Carlo simulations, more than $98 \%$ of neutrons emitted in the ED process were registered.

\section{A. Experimental study of neutron calorimeter}

The neutron zero-degree calorimeter (ZDC) served as a key component of the setup in the ALICE-LUMI experiment. According to results of Monte Carlo simulation studies, the calorimeter has been designed as a periodic structure of 40 modules with the transverse size of $7 \mathrm{~cm} \times 10 \mathrm{~cm}$. Each module consisted of three tungsten slabs of $2.5 \mathrm{~mm}$ in thickness, followed by a 2-mm-thick scintillator slab. The slabs are tilted at an angle of 45 degrees to the beam direction. The light from each scintillator slab was collected by means of two-wavelength-shifter plastic Bicron fibers, which were glued into the grooves on the lateral edges of each scintillator. Opposite sides of optical fibers were coated by aluminum. In this way scintillator light was delivered to a XP2020 photomultiplier.

Several requirements had to be fulfilled concerning the neutron calorimeter. First, the energy resolution for $30-\mathrm{GeV}$ neutrons has to be sufficiently good to discriminate the peak due to $1 n$ emission from the peak due to $2 n$ emission. Second, the response of the neutron calorimeter has to be approximately constant over the calorimeter face area hit by the neutrons.

The assembled calorimeter was tested with a pion beam. The calorimeter response showed a very good linearity as a function of the pion energy ranging from 10 to $100 \mathrm{GeV}$. Gaussian fits to the pulse-height distributions were used with the peak value $\mu$ and its width $\sigma$ as fit parameters. For example, the obtained relative energy resolution $\sigma / \mu$ was $\sim 25 \%$ for $30-\mathrm{GeV}$ pions. In addition, the sensitivity of the calorimeter response to the beam impact point was tested with a $40-\mathrm{GeV}$ proton beam.

\section{B. Processing of experimental data}

We used an average beam intensity of $5 \times 10^{5}$ ions per burst. This sufficiently low beam intensity was chosen to reduce a pileup effect during the spill. A correction for the pileup effect was also taken into account in data analysis. The present data were collected with a $30 A-\mathrm{GeV} \mathrm{Pb}$ beam.

Three types of triggers were used for monitoring and data taking: a random trigger to monitor pedestal behavior and to check noise correlations, a beam trigger with the S0 detector only, and a physics trigger. The physics trigger was fired when the energy deposited in the neutron calorimeter exceeded $8 \mathrm{GeV}$ in coincidence with the signals from the S0 and S1 detectors.

The calorimeter energy scale was determined from the proton peak obtained in a special proton run. The bending magnets (MBPL and MBPS) were switched off during this run.

As discussed in Sec. II A, neutrons from electromagnetic dissociation are emitted very close to beam rapidity. The average energy of such neutrons amounts to $30 \mathrm{GeV}$. Therefore, the validity of the energy calibration of the neutron calorimeter can be independently confirmed by considering energy spectra in $\mathrm{Pb}$ runs. A strong $1 n$ peak and a smaller $2 n$ peak are clearly identified in the energy spectrum shown in Fig. 5. A flat shoulder corresponding to a $3 n$ peak can be also identified. The energy resolution for $30-\mathrm{GeV}$ neutrons, of the order of $25 \%$, is consistent with the test results obtained with pion and proton beams. An empty-target contribution to energy spectra was subtracted from the raw data.

The measured energy spectra were fitted by the sum of Gaussians. The peak values were used as free parameters in the fit. The positions, $\mu_{2 n}$ and $\mu_{3 n}$, and widths, $\sigma_{2 n}$ and $\sigma_{3 n}$, of the Gaussians representing $2 n$ and $3 n$ peaks, respectively, were taken to be fixed. They were calculated from the corresponding values for the $1 n$ peak as $\mu_{2 n}=2 \mu_{1 n}, \sigma_{2 n}=\sqrt{2} \sigma_{1 n}$ and $\mu_{3 n}=$ $3 \mu_{1 n}, \sigma_{3 n}=\sqrt{3} \sigma_{1 n}$. The absolute values of $1 n$ and $2 n$ cross sections were calculated based on the incident $\mathrm{Pb}$ flux given by the S0 detector.

The probability of two sequential $1 n X$ emission events in the 2.94-mm-thick $\mathrm{Pb}$ target was estimated to be 0.089 . Such a process mimics a true $2 n X$ emission event via a pair of independent $1 n X$ events. Therefore, appropriate corrections were applied to the measured rates. For example, the measured number of $1 n X \mathrm{PbPb}$ events was increased by $\sim 10 \%$, whereas the number of $2 n$ events was reduced by $\sim 30 \%$. Corrections of the same kind, but lower in magnitude, were applied to $1 n X$ and $2 n X$ rates on other targets. In addition, $\sim 1-2 \%$ corrections for the secondary hadronic interactions of emitted neutrons in the targets were taken into account.

\section{COMPARISON OF EXPERIMENTAL DATA WITH CALCULATED CROSS SECTIONS}

Calculation results for hadronic and electromagnetic contributions to $1 n$ and $2 n$ emission and obtained experimental data are given in Tables III, IV, and V. The reported experimental errors include systematic and statistical errors. A systematic error of $\sim 8 \%$ is estimated from the uncertainties of the peak heights obtained by the fit of the sum of Gaussians to the neutron energy spectrum. The calculated hadronic contributions present upper limits for hadronic processes registered by the neutron calorimeter. Indeed, the transverse momentum distribution of knock-out nucleons in hadronic collisions is much wider compared to the acceptance of the neutron calorimeter, as discussed in Sec. II B. The calculated ED cross sections are visualized in Fig. 6 along with the measured cross sections. We outline thereafter the reasons 


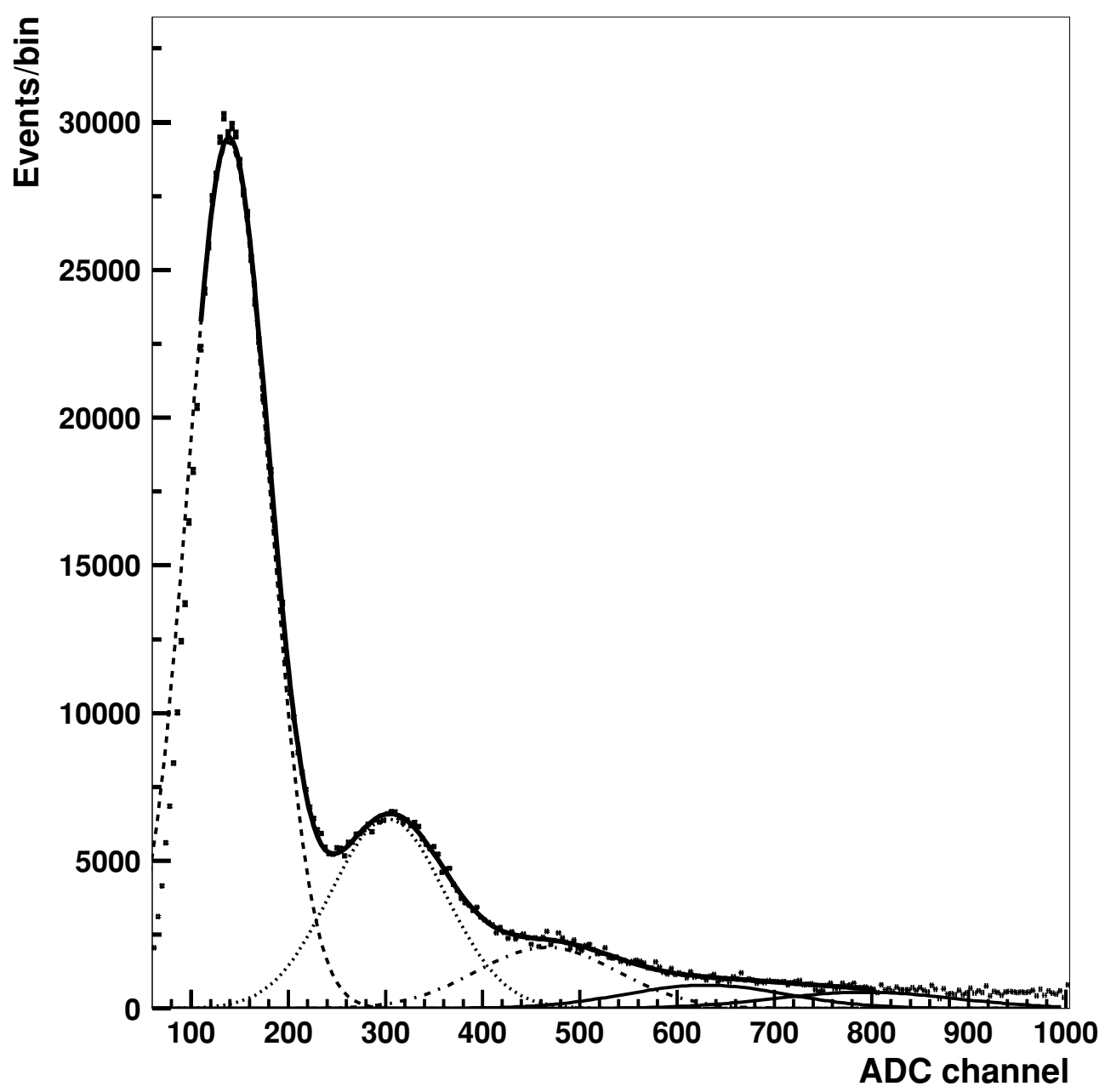

FIG. 5. Energy spectrum of the neutron calorimeter in the Pb run (points) obtained after subtraction of an empty-target contribution. Fit results for the sum of Gaussians are shown by the thick solid line; the results for each individual $1 n, 2 n, 3 n$, and $4 n$ peak are shown by the dashed, dotted, dash-dotted, and thin solid lines, respectively.

to interpret the measured cross sections of forward $1 n$ and $2 n$ emission as cross sections of electromagnetic processes.

First, the dominance of the electromagnetic interaction in $1 n$ and $2 n$ emission in the forward direction is predicted by theory. Indeed, as follows from Tables III and IV, the calculated upper limits for hadronic contributions are typically less than the experimental errors. The exception to this rule is $1 n$ emission in $\mathrm{PbAl}$ collisions with the hadronic contribution estimated to be $\sim 25 \%$ of the experimental cross section and $2 n$ emission in $\mathrm{PbAl}$ and $\mathrm{PbCu}$ collisions with hadronic contributions of $\sim 100 \%$ and $\sim 25 \%$, respectively. However,

TABLE III. Calculated and measured $1 n X$ cross sections (b) for dissociation of $30 A-G e V ~ P b$ on $\mathrm{Al}, \mathrm{Cu}, \mathrm{Sn}$, and $\mathrm{Pb}$ targets.

\begin{tabular}{lccc}
\hline \hline Target & Hadronic & ED (Stat. only) & Experiment \\
\hline $\mathrm{Al}$ & 0.12 & 0.55 & $0.51 \pm 0.05$ \\
$\mathrm{Cu}$ & 0.13 & 2.58 & $2.6 \pm 0.3$ \\
$\mathrm{Sn}$ & 0.14 & 7.33 & $8.8 \pm 0.9$ \\
$\mathrm{~Pb}$ & 0.15 & 18.25 & $18.3 \pm 1.8$ \\
\hline \hline
\end{tabular}

as previously discussed, in these cases most of the neutrons are emitted beyond the acceptance limits of the neutron calorimeter.

Second, the dependence of the measured $1 n X$ and $2 n X$ cross sections on the target charge $Z_{\text {target }}$ indicates the electromagnetic nature of forward neutron emission in $\mathrm{Pb}$ dissociation, as shown in Fig. 6. Both the absolute cross sections $\sigma$ and the values divided by $Z_{\text {target }}^{2}$ are plotted.

In the WW method the flux of equivalent photons as seen by a projectile is proportional to $Z_{\text {target }}^{2}$. Therefore, a very flat dependence of $\sigma / Z_{\text {target }}^{2}$ is expected for electromagnetic

TABLE IV. Calculated and measured $2 n X$ cross sections (b) for dissociation of $30 A-\mathrm{GeV} \mathrm{Pb}$ on $\mathrm{Al}, \mathrm{Cu}, \mathrm{Sn}$, and $\mathrm{Pb}$ targets.

\begin{tabular}{lccc}
\hline Target & Hadronic & ED (Stat. only) & Experiment \\
\hline $\mathrm{Al}$ & 0.13 & 0.09 & $0.13 \pm 0.02$ \\
$\mathrm{Cu}$ & 0.14 & 0.45 & $0.57 \pm 0.07$ \\
$\mathrm{Sn}$ & 0.15 & 1.29 & $2.0 \pm 0.2$ \\
$\mathrm{~Pb}$ & 0.16 & 3.27 & $3.6 \pm 0.4$ \\
\hline \hline
\end{tabular}


TABLE V. Calculated and measured sum of $1 n X$ and $2 n X$ ED cross sections (b) for dissociation of $30 A-\mathrm{GeV} \mathrm{Pb}$ on $\mathrm{Al}, \mathrm{Cu}, \mathrm{Sn}$, and $\mathrm{Pb}$ targets.

\begin{tabular}{lcc}
\hline \hline Target & ED theory (Stat. only) & Experiment \\
\hline $\mathrm{Al}$ & 0.64 & $0.64 \pm 0.05$ \\
$\mathrm{Cu}$ & 3.03 & $3.2 \pm 0.3$ \\
$\mathrm{Sn}$ & 8.62 & $10.8 \pm 0.9$ \\
$\mathrm{~Pb}$ & 21.52 & $21.9 \pm 1.8$ \\
\hline \hline
\end{tabular}

dissociation. Some deviations of $\sigma / Z_{\text {target }}^{2}$ from a constant value exist owing to an additional $Z_{\text {target }}$ dependence of the energy cutoff of the spectrum of equivalent photons. This cutoff value is calculated as $E_{\max }=\gamma / R_{\text {target }}$, where the radius of the target nucleus $R_{\text {target }}$ is also related to $Z_{\text {target }}$. For example, in our calculations $E_{\max }=0.57 \mathrm{GeV}$ for $\mathrm{Pb}$ dissociation on $\mathrm{Al}$, whereas it is $0.42 \mathrm{GeV}$ for $\mathrm{Pb}$ dissociation on $\mathrm{Pb}$. As shown in Fig. 6, this leads to a small increase $(\sim 10 \%)$ in $\sigma / Z_{\text {target }}^{2}$ for light targets.

The measured $1 n X$ cross sections are in good agreement with the values calculated by the RELDIS code. The general trend of the data clearly indicates the electromagnetic nature

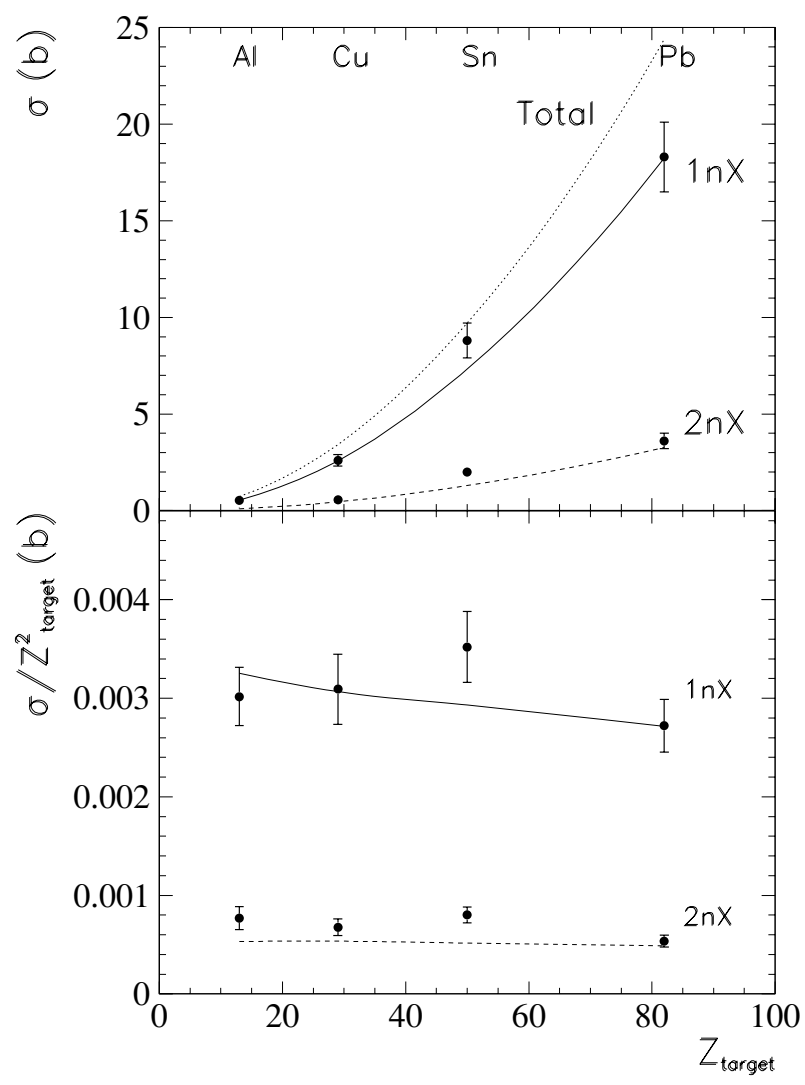

FIG. 6. (Top) Points show measured cross sections of forward $1 n$ and $2 n$ emission in dissociation of $30 A-\mathrm{GeV} \mathrm{Pb}$ ions on $\mathrm{Al}, \mathrm{Cu}$, $\mathrm{Sn}$, and $\mathrm{Pb}$ targets as a function of target charge $Z_{\text {target }}$. Calculated total, $1 n X$, and $2 n X$ ED cross sections are shown by dotted, solid, and dashed lines, respectively. (Bottom) $1 n X$ and $2 n X$ cross sections divided by $Z_{\text {target }}^{2}$. of $\mathrm{Pb}$ dissociation. This confirms the expectations presented in Sec. II A that the hadronic contribution to few-neutron emission is greatly suppressed in our experiment by the acceptance of the neutron calorimeter aimed at the registration of particles with low transverse momentum $P_{t} \leqslant 0.15 \mathrm{GeV} / c$.

As for the $2 n X$ emission, the $Z_{\text {target }}$ dependence also clearly reveals the electromagnetic nature of $\mathrm{Pb}$ dissociation in this case. However, the experimental values are slightly underestimated by theory. This discrepancy between the calculated and experimental $2 n X$ cross sections can be reduced by neglecting the direct nonstatistical contribution in the GDR decay. In Tables III-V the RELDIS results are given for the pure statistical GDR decay via evaporation of neutrons.

The measured sum of $1 n X$ and $2 n X$ cross sections is presented in Table $\mathrm{V}$ and compared to the RELDIS results. This sum is in better agreement with theory because the total $\gamma \mathrm{Pb}$ cross section used in the calculations was measured in experiments with monoenergetic photons [30,31] with reasonable accuracy. Larger uncertainties were connected with the measurements of partial $1 n$ and $2 n$ cross sections, resulting in noticeable disagreement between the measurements performed at Saclay [30] and Livermore [31]. Based on the observation that the total photoneutron yields, $\sigma(\gamma, n)+$ $2 \sigma(\gamma, 2 n)+3 \sigma(\gamma, 3 n)$, obtained in Livermore and Saclay experiments agree well, an explanation for this disagreement was put forward in Ref. [43]. It was concluded that the neutron multiplicity sorting procedure adopted at Saclay was not correct since some $(\gamma, 2 n)$ events were interpreted as pairs of $(\gamma, n)$ events. Recently, in Ref. [44] this finding has received further support through a detailed consideration of photonuclear data for a much wider set of 19 target nuclei.

\section{CONCLUSIONS}

Our experimental data on the forward $1 n$ and $2 n$ emission in the fragmentation of $30-A \mathrm{GeV}{ }^{208} \mathrm{~Pb}$ ions on $\mathrm{Al}, \mathrm{Cu}, \mathrm{Sn}$, and $\mathrm{Pb}$ targets clearly reveal the electromagnetic nature of the process. This is proved by considering the dependence of the cross sections on the target charge.

Our neutron calorimeter was specially designed with a narrow $P_{t}$ acceptance aimed at the registration of neutrons emitted in electromagnetic dissociation. In particular, our design is based on the predictions of the RELDIS model for the transverse-momentum distribution of such neutrons. Our measurements confirm the expectation that the particles produced in peripheral grazing nuclear collisions have a wider $P_{t}$ distribution and have to be strongly suppressed in the present experiment. Indeed, the hadronic contribution to $1 n X$ and $2 n X$ cross sections estimated by the abrasion-ablation model was found to be relatively small, but not negligible compared to the electromagnetic contribution to these channels. This is especially true for $\mathrm{Pb}$ dissociation on $\mathrm{Al}$. However, even in this case the measured cross sections are consistent with the RELDIS model predictions for electromagnetic dissociation.

The dominance of electromagnetic dissociation found in $1 n$ and $2 n$ emission makes the comparison between RELDIS results and experimental data quite straightforward. The measured $1 n X$ cross sections are well described by theory. The 
$2 n X$ cross sections, which are 4-5 times lower than $1 n X$ cross sections, are slightly underestimated by theory. Nevertheless, the sum of $1 n X$ and $2 n X$ cross sections is in good agreement with theory.

The present study has two main outcomes. First, the predictive power of the RELDIS model of electromagnetic dissociation is confirmed. Particularly, this model can be used to calculate the sum of $1 n$ and $2 n$ emission rates in mutual electromagnetic dissociation of ultrarelativistic nuclei. This was proposed in Ref. [9] for the purpose of calibration of the beam luminosity measurements in $\mathrm{PbPb}$ collisions at the LHC. As shown in Ref. [9], the mutual $1 n$ and, especially, $2 n$ rates taken singly can be calculated less accurately.

Second, the obtained experimental data provide a challenge to theory to better describe partial neutron emission cross sections. Currently the model implements the harmonicoscillator picture of multiple excitations and the statistical approach to describe GDR decay. Deviations from this picture have been found at lower heavy-ion energies [45], and such deviations can be also present at $30-A \mathrm{GeV}$. Therefore, more advanced theoretical approaches can be tested with the present data on $1 n$ and $2 n$ emission cross sections.

\section{ACKNOWLEDGMENTS}

We would like to thank the ion source and accelerator teams of CERN for the good performance of the $\mathrm{Pb}$ beam during data taking. I.A.P. thanks V. V. Varlamov for fruitful discussions. This work was supported by the Russian Foundation for Basic Research, Grant 02-02-16013.
[1] T. J. Hallman, D. E. Kharzeev, J. T. Mitchell, and T. S. Ullrich (eds.), Proceedings of the 15th International Conference on Ultrarelativistic Nucleus Nucleus Collisions QM2001, Stony Brook, USA, January 15-20, 2001, Nucl. Phys. A698, 3 (2002).

[2] H. Gutbrod, J. Aichelin, and K. Werner (eds.), Proceedings of the 16th International Conference on Ultrarelativistic Nucleus Nucleus Collisions QM2002, Nantes, France, July 18-24, 2002, Nucl. Phys. A715, 3 (2003).

[3] S. A. Bass, B. Mueller, G. S. F. Stephans, and T. Ullrich (eds.), Proceedings of the 7th International Conference on Strangeness in Quark Matter SQM2003, Atlantic Beach, USA, March 12-17, 2003, J. Phys. G: Nucl. Part. Phys. 30, S1 (2004).

[4] F. Krauss, M. Greiner, and G. Soff, Prog. Part. Nucl. Phys. 39, 503 (1997).

[5] G. Baur, K. Hencken, and D. Trautmann, J. Phys. G: Nucl. Part. Phys. 24, 1657 (1998).

[6] A. J. Baltz, C. Chasman, and S. White, Nucl. Instrum. Methods Phys. Res. A417, 1 (1998).

[7] G. Baur, K. Hencken, D. Trautmann, S. Sadovsky, and Y. Kharlov, Phys. Rep. 364, 359 (2002).

[8] I. A. Pshenichnov, I. N. Mishustin, J. P. Bondorf, A. S. Botvina, and A. S. Iljinov, Phys. Rev. C 57, 1920 (1998).

[9] I. A. Pshenichnov, J. P. Bondorf, I. N. Mishustin, A. Ventura, and S. Masetti, Phys. Rev. C 64, 024903 (2001).

[10] G. Baur, K. Hencken, A. Aste, D. Trautmann, and S. R. Klein, Nucl. Phys. A729, 787 (2003).

[11] A. J. Baltz, M. J. Rhoades-Brown, and J. Weneser, Phys. Rev. E 54, 4233 (1996).

[12] S. R. Klein, Nucl. Instrum. Methods Phys. Res. A459, 51 (2001).

[13] M. Gallio, W. Klempt, L. Leistam, J. De Groot, and J. Schükraft, ALICE: Technical Design Report of the Zero Degree Calorimeter (ZDC), ALICE-TDR-3, CERN-LHCC-99-005.

[14] I. A. Pshenichnov, J. P. Bondorf, A. B. Kurepin, I. N. Mishustin, and A. Ventura, ALICE Internal Note, ALICE-INT-2002-07, https://edms.cern.ch/document/339134.

[15] C. Oppedisano, ALICE Internal Note, ALICE-INT-2002-08, https://edms.cern.ch/document/339254.

[16] T. Aumann, J. V. Kratz, E. Stiel, K. Sümmerer, W. Brüchle, M. Schädel, G. Wirth, M. Fauerbach, and J. C. Hill, Phys. Rev. C 47, 1728 (1993).
[17] J. C. Hill, A. Petridis, B. Fadem, and F. K. Wohn, Nucl. Phys. A661, 313 (1999).

[18] M. Chiu, A. Denisov, E. Garcia, J. Katzy, A. Makeev, M. Murray, and S. White, Phys. Rev. Lett. 89, 012302 (2002).

[19] S. Datz, J. R. Beene, P. Grafström, H. Knudsen, H. F. Krause, R. H. Schuch, and C. R. Vane, Phys. Rev. Lett. 79, 3355 (1997).

[20] P. Grafström, S. Datz, H. F. Krause, C. R. Vane, H. Knudsen, U. Mikkelsen, R. H. Schuch, C. Scheidenberger, and Z. Z. Vilakazi, CERN-SL-99-033-EA, Proceedings of the 1999 Particle Accelerator Conference, New York, 29 March-2 April 1999, vol. 3, 1671 (1999).

[21] H. Dekhissi, G. Giacomelli, M. Giorgini, S. Manzoor, L. Patrizii, V. Popa, P. Serra, and V. Togo, Nucl. Phys. A662, 207 (2000).

[22] S. Cecchini, G. Giacomelli, M. Giorgini, G. Mandrioli, L. Patrizii, V. Popa, P. Serra, G. Sirri, and M. Spurio, Nucl. Phys. A707, 513 (2002).

[23] C. Scheidenberger, I. A. Pshenichnov, T. Aumann, S. Datz, K. Sümmerer, J. P. Bondorf, D. Boutin, H. Geissel, P. Grafström, H. Knudsen, H. F. Krause, B. Lommel, S. P. Moller, G. Münzenberg, R. H. Schuch, E. Uggerhøj, U. Uggerhøj, C. R. Vane, A. Ventura, Z. Z. Vilakazi, and H. Weick, Phys. Rev. Lett. 88, 042301 (2002).

[24] W. J. Llope and P. Braun-Munzinger, Phys. Rev. C 41, 2644 (1990).

[25] I. A. Pshenichnov, I. N. Mishustin, J. P. Bondorf, A. S. Botvina, and A. S. Iljinov, Phys. Rev. C 60, 044901 (1999).

[26] B. L. Berman and S. C. Fultz, Rev. Mod. Phys. 47, 713 (1975).

[27] B. L. Berman, R. E. Pywell, S. S. Dietrich, M. N. Thompson, K. G. McNeill, and J. W. Jury, Phys. Rev. C 36, 1286 (1987).

[28] A. S. Iljinov, I. A. Pshenichnov, N. Bianchi, E. De Sanctis, V. Muccifora, M. Mirazita, and P. Rossi, Nucl. Phys. A616, 575 (1997).

[29] J. P. Bondorf, A. S. Botvina, A. S. Iljinov, I. N. Mishustin, and K. Sneppen, Phys. Rep. 257, 133 (1995).

[30] A. Veyssière, H. Beil, R. Bergère, P. Carlos, and A. Leprêtre, Nucl. Phys. A159, 561 (1970).

[31] R. R. Harvey, J. T. Caldwell, R. L. Bramblett, and S. C. Fultz, Phys. Rev. B 136, 126 (1964).

[32] S. N. Belyaev and V. A. Semenov, Bull. Acad. Sci. USSR, Phys. Ser. 55 (5), 66 (1991). 
[33] V. V. Varlamov, N. G. Efimkin, B. S. Ishkhanov, and V. V. Sapunenko, Yadernye Konstanty 1, 52 (1993).

[34] R. F. Askew and A. P. Batson, Nucl. Phys. 20, 408 (1960).

[35] F. Tagliabue and J. Goldemberg, Nucl. Phys. 23, 144 (1961).

[36] R. Alarcon, P. L. Cole, D. S. Dale, P. T. Debevec, and L. J. Morford, Phys. Rev. C 43, R2470 (1991).

[37] J. Hüfner, K. Schäfer, and B. Schürmann, Phys. Rev. C 12, 1888 (1975).

[38] L. F. Oliveira, R. Donangelo, and J. O. Rasmussen, Phys. Rev. C 19, 826 (1979).

[39] J.-J. Gaimard and K. H. Schmidt, Nucl. Phys. A531, 709 (1991).

[40] M. de Jong, K.-H. Schmidt, B. Blank, C. Böckstiegel, T. Brohm, H.-G. Clerc, S. Czajkowski, M. Dornik, H. Geissel, A. Grewe, E. Hanelt, A. Heinz, H. Irnich, A. R. Junghans, A. Magel, G. Münzenberg, F. Nickel, M. Pfützner, A. Piechaczek,
C. Scheidenberger, W. Schwab, S. Steinhäuser, K. Sümmerer, W. Trinder, B. Voss, and C. Ziegler, Nucl. Phys. A628, 479 (1998).

[41] J. Benlliure, K. H. Schmidt, D. Cortina-Gil, T. Enqvist, F. Farget, A. Heinz, A. R. Junghans, J. Pereira, and J. Taieb, Nucl. Phys. A660, 87 (1999).

[42] P. Senger and H. Ströbele, J. Phys. G: Nucl. Part. Phys. 25, R59 (1999).

[43] E. Wolynec, A. R. V. Martinez, P. Gouffon, Y. Miyao, V. A. Serrao, and M. N. Martins, Phys. Rev. C 29, 1137 (1984).

[44] V. V. Varlamov, N. N. Peskov, D. S. Rudenko, and M. S. Stepanov, Preprint SINP MSU 2003-2/715.

[45] LAND collaboration, K. Boretzky et al., Phys. Rev. C 68, 024317 (2003). 